\title{
Perfil dos Observatórios de Turismo da Rede Brasileira de Observatórios de Turismo
}

\section{Profile of the Tourism Observatories of the Brazilian Network of Tourism Observatories}

\author{
João Evangelista Dias Monteiro. \\ Osiris Marques. \\ Giovanna Tavares. \\ Letícia Santos-Silva. \\ Valério Souza-Neto.
}

\begin{abstract}
RESUMO
Estruturar, analisar e disponibilizar dados estatísticos constitui-se num pré-requisito para uma gestão baseada em evidências técnicas e científicas de destinos turísticos. Nesta perspectiva, a Rede Brasileira de Observatórios de Turismo (RBOT), rede que reúne atualmente 58 observatórios de turismo em todo território nacional, tem desempenhado um papel importante no fomento à utilização de pesquisas e dados estatísticos para a orientação do planejamento e do desenvolvimento sustentável do turismo no Brasil. O presente estudo técnico tem como objetivo a realização do levantamento de informações sobre a composição, a estrutura e a dinâmica das pesquisas realizadas pelos observatórios membros da RBOT. Para tal, realizou-se uma pesquisa survey, aplicada em 2021, com 45 representantes dos observatórios de turismo. Os resultados indicaram que a maioria dos respondentes reconhecem a importância da RBOT no crescimento e consolidação dos observatórios. Em relação à parte operacional, o destaque positivo foi a constatação da presença dos turismólogos em quase todos os observatórios. Entre as preocupações estão a dificuldade orçamentária para a execução das pesquisas e a limitação de equipe dos observatórios. Conclui-se que apesar do avanço nos últimos anos, os observatórios ainda têm muitos desafios no processo de consolidação no monitoramento do crescimento e desenvolvimento do turismo sustentável no Brasil.
\end{abstract}

Palavras-chave: Rede Brasileira de Observatórios de Turismo. RBOT. Perfil dos observatórios. Gestão baseada em evidências

\begin{abstract}
Structuring, analyzing and making available statistical data is a prerequisite for a technical and scientific evidence-based management of tourist destinations. In this perspective, the Brazilian Network of Tourism Observatories (RBOT), a network that currently brings together 58 tourism observatories throughout the national territory, has played an important role in promoting the use of research and statistical data in guiding the planning of sustainable tourism development in Brazil. This technical study aims to carry out a survey of information on the composition, structure and dynamics of research carried out by observatories, members of RBOT. To this end, a survey research was carried out, applied in 2021, with 45 representatives of tourism observatories. The results indicated that most respondents recognize the importance of RBOT in the growth and consolidation of observatories. Regarding the operational part, the positive highlight was the finding of the presence of tourism specialists in almost all observatories.
\end{abstract}


Among the concerns are budgetary difficulties for carrying out the surveys and the limited staff of the observatories. It is concluded that despite advances in recent years, observatories still face many challenges in the process of consolidating the monitoring of the growth and development of sustainable tourism in Brazil.

Keywords: Brazilian Network of Tourism Observatories. RBOT. Profile of observatories. Evidencebased management

\section{INTRODUÇÃO}

Turistas produzem (XIANG, 2018) e consomem uma variedade de dados não estruturados que impactam na tomada de decisão dos mesmos (XIANG et al., 2014). Estruturar, analisar e disponibilizar esses dados constitui-se como um cenário de muita utilidade para os destinos turísticos (CACHO et al., 2016). Os dados não permitem apenas que as empresas tomem decisões baseadas em informações (apoiar a gestão baseada em evidências, simplificar as complexidades administrativas e identificar novas oportunidades de negócios), mas também são o 'óleo' das empresas da nova economia para construir novos modelos de negócios para monetizar os recursos de informação (SIGALA, 2018).

Nos últimos anos identificamos esforços nas mais diversas escalas para a produção de gestão do turismo baseada em evidências científicas e dados estatísticos. Reconhecendo a importância dos observatórios na produção de dados e estatísticas para o monitoramento do desenvolvimento sustentável do turismo, a Organização Mundial do Turismo (OMT) criou em 2004, a Rede Internacional de Observatórios De Turismo Sustentável (INSTO) (OMT, s.a.). Em nível nacional, percebe-se atenção dada pelo Ministério do Turismo (MTUR) em seu Plano Nacional de Turismo (2018-2022), ao qual destaca a importância do fortalecimento da Rede Brasileira dos Observatórios de Turismo, além da ampliação de investimentos para realização de pesquisas e estudos para entendimento das dinâmicas do fenômeno turístico (MTUR, 2018).

Neste panorama encontra-se a Rede Brasileira de Observatórios de Turismo (RBOT), iniciada em 2016 (ALVARES; SANTOS; PERINOTTO, 2020) e criada em 11 de maio de 2017, no Encontro Paranaense dos Observatórios de Turismo, mediante assinatura da "Carta Curitiba" (MARQUES, 2017). A RBOT "reúne observatórios de turismo em todo território nacional com o intuito de fomentar o turismo planejado e 
gerido com base em dados em nosso país" (MARQUES; MONTEIRO; SOUZA NETO, 2021, s.p.), e possui, atualmente, 58 (cinquenta e oito) observatórios membros, com representação macrorregional equilibrada em todas as regiões do Brasil.

Pela sua alta representatividade em território nacional e vasta heterogeneidade entre os estados e municípios brasileiros e suas formas de gestão do turismo nas mais diversas escalas, propomos, por meio deste artigo técnico, realizar o levantamento de informações sobre a composição, a estrutura e a dinâmica das pesquisas realizadas pelos observatórios membros da RBOT. Para tal, realizou-se uma pesquisa survey (FREITAS et al., 2000) com amostragem por conveniência de 45 (quarenta e cinco) membros da Rede Brasileira de Observatórios de Turismo.

O presente artigo estrutura-se da seguinte maneira: i. Breve introdução do tema de estudo; ii. Um breve histórico sobre a Rede Brasileira de Observatórios de Turismo; iii. Procedimentos metodológicos aplicados para realização do objetivo da pesquisa; iv. Resultados descritivos dos dados coletados; e por fim, v. as Considerações finais.

\section{HISTÓRICO DA REDE BRASILEIRA DE OBSERVATÓRIOS DE TURISMO}

A Rede Brasileira de Observatórios de Turismo (RBOT) iniciou-se em 2016 (ALVARES; SANTOS; PERINOTTO, 2020) tendo sida oficializada em 2017, no Encontro Paranaense dos Observatórios de Turismo, realizado em Curitiba (Paraná), no dia 11 de maio de 2017, e contou com a presença de 12 (doze) observatórios brasileiros e 3 (três) observatórios internacionais. Neste dia, os observatórios do Paraná, do Espírito Santo, do Rio de Janeiro (UFF), do Distrito Federal, de Maringá, de São Paulo, de Foz do Iguaçu, de Goiás, de Minas Gerais, de Bonito, e de Campo Grande, assinaram a "Carta de Curitiba de apoio à criação da Rede Brasileira de Observatórios de Turismo", que tinha como objetivo "promover a integração e o compartilhamento de dados e a uniformização de metodologias de coleta, tratamento, análise, geração e monitoramento de modo a contribuir para o progresso do turismo no país" (RBOT, 2017) e contou com o apoio de membros de diversas entidades, como o Ministério do Turismo, Secretaria Nacional de Turismo do Paraguai, Sebrae, Fecomércio, Convention 
\& Visitors Bureau, Agência Valenciana de Turismo, entre outras entidades de escalas menores.

Após a criação da RBOT, pouco mais de 5 (cinco) meses após o Encontro Paranaense, em uma quarta-feira (01 de novembro de 2017), aconteceu o I Encontro da Rede Brasileira de Observatórios de Turismo, no Museu das Minas e do Metal - MM Gerdau, em Belo Horizonte (Minas Gerais) (BH EVENTOS, 2017). Este encontro, organizado no formato de oficinas, teve como objetivo a análise da situação das pesquisas sobre Destinos Turísticos Inteligentes no Brasil sob a coordenação do professor e pesquisador José Gândara (Observatório de Turismo da Universidade Federal do Paraná), além da apresentação das pesquisas sobre a situação dos observatórios de turismo no Brasil, ministrada pelo Ítalo Dantas, representante do Ministério do Turismo. (SECULT-MG, 2017).

O segundo encontro da RBOT ocorreu em Foz do Iguaçu, no polo tecnológico de Itaipu (Paraná), nos dias 08 e 09 de maio de 2018 com o intuito de discutir "temas pertinentes à operação e à melhoria das ações dos Observatórios, para que se tornem mais dinâmicos, conectados e inovadores" (RBOT, 2018). Neste encontro foi possível contar com a presença de 26 observatórios de turismo de todo o país. De todos os observatórios que se [tinha] conhecimento e foram convidados, apenas dois (Bahia e Ponta Grossa) não puderam estar presentes" (RBOT, 2018). A relevância da Rede aumentava cada vez mais e neste encontro destacaram a presença da Associação Nacional de Pesquisa e Pós-Graduação em Turismo (ANPTUR).

Em 30 de outubro de 2019 foi realizado o terceiro encontro da RBOT, em Belo Horizonte, que fez parte da programação do III Seminário Mineiro de Pesquisa e Inovação em Turismo. Neste encontro definiu-se pesquisas e indicadores recomendados a todos os observatórios. Para sistematizar as atividades da RBOT foram criados grupos de trabalho por tema: i. elaboração de protocolos e padronização de instrumentos de pesquisa e ii. articulação entre grupos de pesquisa de turismo. Adicionalmente, discutiuse o papel dos observatórios de turismo na busca dos Objetivos de Desenvolvimento Sustentável, da Organização Mundial das Nações Unidas. Em específico, em relação aos indicadores propostos pela International Network of Sustainable Tourism Observatories (INSTO). 
Durante a pandemia da Covid-19, que restringiu a mobilidade de todos e impactou significativamente o turismo (COELHO; MAYER, 2020; MAYER, COELHO, 2021), entre 08 de abril a 27 de abril de 2020, os observatórios membros da RBOT realizaram uma macro pesquisa de Sondagem Empresarial dos Impactos da Covid-19 no Turismo. A pesquisa teve 4.921 empresas respondentes de vários estados do Brasil e foi importante para o entendimento e dimensionamento dos impactos da pandemia nos negócios das atividades características de turismo e, por conseguinte, auxiliar na definição de políticas de mitigação dos impactos negativos dos mesmos (MEDAGLIA; SILVEIRA, 2020).

O $4^{\circ}$ Encontro da Rede Brasileira dos Observatórios de Turismo "Pesquisa em Rede: Entrelaçamentos do Turismo Brasileiro", ocorreu entre os dias 08 e 09 de julho de 2020, em ambiente virtual, em decorrência da pandemia da Covid-19. O encontro fora organizado pelo Observatório de Turismo do Estado de Goiás. e contou com a participação de 37 observatórios. (RBOT, 2020).

O V Encontro, também de forma virtual, ocorreu entre 16 e 17 de agosto de 2021, desta vez organizado pelo Observatório de Turismo do Paraná, contou com o apoio do Observatório de Turismo do Estado de Goiás, e teve a participação de 57 observatórios. As palestras foram transmitidas pelo Youtube, onde mais de 7.000 pessoas assistiram.

Pode-se observar que, desde a criação da RBOT, o número de observatórios filiados cresceu significativamente, passando de 12 observatórios no encontro de 2016, que oficializaram seu processo de criação, para 57, no V encontro, realizado em 2021.

Assim, dada a pluralidade e a natureza diversa dos observatórios de Turismo que compõem a RBOT, esta pesquisa tem como objetivo conhecer a estrutura e a dinâmica operacional dos observatórios, além da composição das pesquisas realizadas.

\section{METODOLOGIA}

O método de pesquisa adotado para o presente estudo foi a survey, sendo esta, uma "obtenção de dados ou informações sobre características, ações, ou opiniões de 
pessoas, indicado como representante de uma população-alvo" (FREITAS et al., 2000, p. 105). Como a pesquisa tem como objeto de análise a Rede Brasileira dos Observatórios de Turismo, a população é restrita aos 58 observatórios, atualmente, membros da RBOT.

A coleta de dados deu-se em dois momentos, sendo o primeiro realizado entre 30 (trinta) de junho a 14 (quatorze) de julho de 2021 e o segundo momento realizado entre 12 (doze) a 15 (quinze) de outubro de 2021. Tendo sido realizada pela plataforma do Google Forms e disseminadas no grupo do WhatsApp da RBOT. As pesquisas em ambiente online são flexíveis, têm um alcance global, permitem a conclusão em tempo hábil, com fácil entrada de dados (MAIR, 2011), e constitui-se como uma metodologia amplamente adotada no campo dos estudos de Turismo (HUNG; LAW, 2011), sendo apropriada para uma pesquisa que abarca um país de proporções continentais como o Brasil.

O questionário possuía 14 (quatorze) perguntas para compreensão da estrutura, situação, pesquisas realizadas e formas de divulgação dos observatórios. As perguntas realizadas pela presente pesquisa encontram-se na tabela 1 .

Dos 58 (cinquenta e oito) observatórios membros da RBOT, 49 (quarenta e nove) responderam à pesquisa, correspondendo assim a $84,48 \%$ da população da rede, sendo considerada uma amostra representativa para perfilamento dos observatórios de turismo do Brasil e compreensão da realidade dos mesmos. Para análise, excluímos 4 (quatro) respostas (uma por estar em construção, duas por estarem inativas e a terceira por ser de uma organização que subsidia a construção de outros observatórios), restando assim um total de $45(77,5 \%)$ respostas analisadas.

Tabela 1: Lista de perguntas do survey online.

\begin{tabular}{ll}
\hline & Pergunta \\
\hline 1. & O ano da criação do Observatório \\
2. & Instituição que dirige o Observatório \\
3. & Formação da equipe dos observatórios \\
4. & Se a equipe do observatório era suficiente
\end{tabular}


5. Como os observatórios suprem essa carência em caso de insuficiência de equipe

6. Pesquisas realizadas regularmente

7. Indicadores monitorados regularmente

8. Realidade orçamentária

9. Principal fonte de recursos do observatório

10. Se o observatório possui site para divulgação de pesquisa

11. Se o observatório possui redes sociais

12. Em quais redes sociais os observatórios possuem presença online

13. Formas de divulgação das pesquisas

14. A relevância da Rede Brasileira dos Observatórios de Turismo para a consolidação e crescimento do observatório

Fonte: Autores.

Para gestão e análise desses dados, os mesmos foram tabulados no Microsoft Excel (RASOOLIMANESH et al., 2021) e serão apresentados por estatísticas descritivas na seção a seguir.

\section{RESULTADOS}

A primeira pergunta do nosso survey diz respeito ao ano de criação dos observatórios. Identifica-se um intervalo de 18 anos (2003-2021) entre a criação do observatório até o atual momento (tabela 1). Estudos qualitativos com os representantes destes observatórios poderão sanar as seguintes questões: se sempre se identificaram como observatórios ou se estão associando a núcleos de pesquisa dentro de órgãos como observatórios em suas respostas.

Ainda se percebe um crescimento lento em comparação à necessidade de tomadas de decisões otimizadas baseadas em dados. Em nossos dados, o ano de 2017 teve o maior número de criação de observatórios, possivelmente derivado da articulação pós-criação da Rede Brasileira de Observatórios de Turismo, que teve o seu movimento de iniciação em 2016 (ALVARES; SANTOS; PERINOTTO, 2020). O segundo ano de maior criação é o ano de 2019, ano posterior à divulgação do Plano Nacional de Turismo previsto para 2018-2022 (MTUR, 2018), ao qual reforça a necessidade dos observatórios e da rede interinstitucional entre os mesmos. 
Tabela 1: Qual é o ano da criação do Observatório?

\begin{tabular}{lll}
\hline Ano de criação & Quantidade & $\%$ \\
\hline 2021 & 2 & $4 \%$ \\
2020 & 3 & $7 \%$ \\
2019 & 6 & $13 \%$ \\
2018 & 4 & $9 \%$ \\
2017 & 9 & $20 \%$ \\
2016 & 3 & $7 \%$ \\
2015 & 3 & $7 \%$ \\
2014 & 1 & $2 \%$ \\
2013 & 4 & $9 \%$ \\
2012 & 3 & $7 \%$ \\
2011 & 2 & $4 \%$ \\
2009 & 1 & $2 \%$ \\
2008 & 2 & $4 \%$ \\
2005 & 1 & $2 \%$ \\
2003 & 1 & $2 \%$ \\
Total & 45 & $100 \%$ \\
\hline
\end{tabular}

Fonte: Autores (2021).

A tabela 2 nos mostra a qual instituição os observatórios de turismo estão vinculados. Deste modo, torna-se evidente a importância da gestão pública (44\%), constituída pelas secretarias estaduais e municipais e Instituições de Ensino (36\%), majoritariamente de ensino público ${ }^{1}$ para a produção de dados confiáveis e relevantes para o turismo. Adicionalmente, os dados demonstram a heterogeneidade ao qual os observatórios são criados. Pode-se identificar observatórios geridos por entidades de classe como a ABBTUR, Abrasel e Fecomércio, assim como empresas mistas, Convention Bureaus, autarquia e associação comercial. Ainda assim, conforme a tabela nos mostra, os dados do turismo necessitam de investimento público para que possam ser estruturados e apropriadamente divulgados.

\footnotetext{
${ }^{1}$ Somente uma instituição sinalizou fazer parte do Ensino Privado.
} 
Tabela 2: Qual é a Instituição que dirige o Observatório?

\begin{tabular}{lll}
\hline Instituição dirigente & Quantidade & $\%$ \\
\hline Associação comercial & 1 & $2 \%$ \\
Autarquia & 1 & $2 \%$ \\
Convention Bureau & 2 & $4 \%$ \\
Empresa mista & 2 & $4 \%$ \\
Entidade de classe & 3 & $7 \%$ \\
Instituição de Ensino & 16 & $36 \%$ \\
Secretaria Estadual & 12 & $24 \%$ \\
Secretaria Municipal & 9 & $20 \%$ \\
Total & 45 & $100 \%$ \\
\hline
\end{tabular}

Fonte: Autores (2021).

Além da importância de orçamento público para a produção de dados e manutenção desses órgãos, quando se analisa os profissionais que fazem parte dos observatórios, identificamos o papel crucial (ênfase nossa) do turismólogo na gestão e operacionalização destes instrumentos de apoio à gestão do turismo. Dos 45 observatórios analisados, 44 (98\%) possuem turismólogos em suas equipes (tabela 3). Além destes, estagiários são elencados em segundo lugar, estando presente em $44 \%$ dos observatórios, seguido por administradores (29\%) e designers (24\%). Torna-se interessante identificar este profissional, o designer (em quarto lugar), pois possui alta relevância como criador de materiais de fácil leitura para o público-alvo das pesquisas, já que dados abertos e conhecimento compartilhado servem como alicerces para a experiência turística e para novos mecanismos de inovação (XIANG, 2018).

Tabela 3: Quais profissionais fazem parte da Equipe do Observatório?

\begin{tabular}{lll}
\hline Formação & Quantidade & \% \\
\hline Turismólogo & 44 & $98 \%$ \\
Estagiário & 20 & $44 \%$ \\
Administrador & 13 & $29 \%$ \\
Designer & 11 & $24 \%$
\end{tabular}




$\begin{array}{lll}\text { Estatístico } & 9 & 20 \% \\ \text { Economista } & 8 & 18 \% \\ \text { Jornalista } & 8 & 18 \% \\ \text { Geógrafo } & 5 & 11 \% \\ \text { Cientista de dados } & 3 & 7 \% \\ \text { Outros }^{2} & 10 & 22 \%\end{array}$

Fonte: Autores (2021).

Em relação à suficiência da equipe para suprir as demandas de pesquisa dos observatórios, 3/4 (76\%) dos observatórios pontuaram que há insuficiência de recursos humanos para lidar com o corpo de trabalho dos observatórios (Tabela 4). Como tentativa de suprir essa carência, os observatórios majoritariamente realizam parcerias (20\%), terceirizam serviços de coleta de dados (20\%) ou contratam consultorias (18\%).

Tabela 4: Respostas das perguntas 4 e 5 - A equipe é suficiente para atender as demandas de pesquisa do Observatório? E Se respondeu não na pergunta anterior, o que o Observatório faz para suprir essa carência?

\begin{tabular}{lll}
\hline Equipe é suficiente & Quantidade & \% \\
\hline Não & 34 & $76 \%$ \\
Sim & 11 & $24 \%$ \\
& & \\
Meios para suprir carência & Quantidade & $\%$ \\
Parceria & 9 & $20 \%$ \\
Terceirização do serviço de coleta de dados & 9 & $20 \%$ \\
Contratação de consultoria & 8 & $18 \%$ \\
Nada & 4 & $9 \%$ \\
Contrata funcionário temporário & 3 & $7 \%$ \\
Voluntariado & 3 & $7 \%$ \\
Bolsista & 2 & $4 \%$ \\
Outros & & $9 \%$ \\
\hline
\end{tabular}

\footnotetext{
${ }^{2}$ Outros inclui: Advogado; Analista e Desenvolvedor de Sistemas; Bióloga; Contratação temporária; Escriturária; Historiador; Hoteleiro; Profissional de Marketing; Tradutor; Voluntário.

${ }^{3}$ Outros incluem: Contratação de serviços especializados sob demanda; Edital; Pesquisa realizada por outro órgão; Trabalha com dados secundários.
} 
Fonte: Autores (2021).

Pontua-se ainda que, ao cruzar os dados das instituições dirigentes dos observatórios (tabela 2) à questão da suficiência de equipe, destes 34 observatórios que elencaram não possuir equipe própria, $27(79,4 \%)$ são de Instituições de Ensino públicas e da gestão pública estadual e municipal. Além disso, dos 32 observatórios que alegaram não possuir orçamento próprio (tabela 8), 26 (81,2\%) são das instituições supramencionadas. Ou seja, pesquisas produzidas por $80 \%$ dos observatórios de turismo estão em condições altamente deficitárias, resultando em uma subutilização destes instrumentos de apoio à gestão e planejamento.

Uma relação interessante que se pode observar entre a suficiência de equipe e o ano de criação dos observatórios é que os observatórios mais recentes possuem equipes capazes de sanar as demandas de pesquisa. Mais especificamente, os observatórios criados até 2014, 13\% destes possuem equipes suficientes, em comparação aos observatórios de 2015 até o presente momento, que 31\% deles possuem capacidade técnicas para sanar as demandas dos observatórios (tabela 5).

Tabela 5: Relação entre ano de criação dos observatórios e suficiência de equipes.

\begin{tabular}{llcl}
\hline Ano de criação & \multicolumn{3}{l}{ Suficiência de equipe } \\
\hline \multirow{2}{*}{ Até 2014} & Não & Sim & Total Geral \\
$2015-2021$ & $88 \%$ & $13 \%$ & $100 \%$ \\
Total Geral & $69 \%$ & $31 \%$ & $100 \%$ \\
\hline
\end{tabular}

Fonte: Autores (2021).

As pesquisas produzidas pelos observatórios variam, mas pode-se observar que existe um núcleo de pesquisas que são realizadas pela maioria dos observatórios: pesquisa de demanda turística, realizada por mais de 3 quartos dos observatórios (78\%), inventário da oferta (53\%), sondagens empresariais e pesquisas de impacto econômico do Turismo (ambos com 51\%) e em eventos (47\%).

Tabela 6: Quais são as pesquisas realizadas pelo Observatório? 


\begin{tabular}{lll}
\hline Pesquisas realizadas & Quantidade & \% \\
\hline Pesquisa de demanda turística & 35 & $78 \%$ \\
Pesquisa de Oferta (Inventário) & 24 & $53 \%$ \\
Pesquisa de Conjuntura (sondagem empresarial) & 23 & $51 \%$ \\
Pesquisa de Impacto Econômico do Turismo & 23 & $51 \%$ \\
Pesquisa de Impacto Econômico em evento & 21 & $47 \%$ \\
Pesquisa de Oferta (Cadeia produtiva) & 17 & $38 \%$ \\
Pesquisa de Opinião Pública & 3 & $7 \%$ \\
Pesquisa em hotéis (Censos, Fluxo de ocupação, Fluxo de & 3 & $7 \%$ \\
Turista) & 2 & $4 \%$ \\
Análise de redes sociais & 2 & $4 \%$ \\
Pesquisa de políticas públicas & 13 & $29 \%$ \\
Outras & &
\end{tabular}

Fonte: Autores (2021).

Destas pesquisas, mais da metade dos observatórios tendem a coletar os indicadores relacionados a perfil do turista (64\%), empregos no setor turístico (62\%), fluxo de passageiros aéreos (60\%), ocupação hoteleira (58\%), gasto médio e permanência média (ambos com 51\%).

Tabela 7: Quais os indicadores do Turismo monitorados?

\begin{tabular}{lll}
\hline Indicadores & Quantidade & \% \\
\hline Perfil do Turista & 29 & $64 \%$ \\
Emprego no turismo & 28 & $62 \%$
\end{tabular}

\footnotetext{
${ }^{4}$ Outras inclui: Análise de ACTs; Atuação dos profissionais; Mercado de trabalho; Perfil de Turistas; Pesquisa de benchmarking (cicloturismo); Pesq. de comunicação; Pesq.de dados móveis; Pesq. de Fluxo Turístico; Pesq. de percepção e de inovação; Pesq. de público em circuitos culturais; Pesq. em eventos; Pesq. mercadológica para atender as demandas dos Turismólogos; Planejamento Estratégico.
} 


\section{ReBOT UFN}

Fluxo de passageiros do aéreo

27

Ocupação hoteleira

$$
26
$$

Gasto Médio

$$
23
$$

Permanência Média

$$
23
$$

21

Número de visitantes do destino

Fluxo de passageiros do rodoviário

Impacto de eventos

20

19

19

18

16

9

9

Preços no turismo

Outros $^{5}$
$60 \%$

$58 \%$

$51 \%$

$51 \%$

$47 \%$

$44 \%$

$42 \%$

$42 \%$

$40 \%$

$36 \%$

$20 \%$

$20 \%$

$16 \%$

Fonte: Autores (2021).

A maioria dos observatórios não possuem orçamento próprio (69\%), como indica a tabela 8. A principal fonte são os recursos próprios ${ }^{6}$ (47\%), seguido por pesquisas e projetos (29\%) e editais, licitações e demais contratações com órgãos públicos (20\%) (tabela 8). Em menor número, há a opção de parcerias com entidades nacionais e internacionais, fator que indica a necessidade de haver uma maior atenção e busca por colaborações e cooperações com entidades dentro e fora do país.

Tabela 8: Respostas das perguntas 8 e 9 - O Observatório tem orçamento próprio? E Qual é a principal fonte de recursos do Observatório?

\begin{tabular}{lll}
\hline Orçamento próprio & Quantidade & $\%$ \\
\hline Não & 31 & $69 \%$ \\
Sim & 14 & $31 \%$ \\
Total & 45 & $100 \%$
\end{tabular}

\footnotetext{
${ }^{5}$ Outros inclui: Atendimentos a turistas; Avaliação qualitativa da infraestrutura urbana; Cadastur; Empresas relacionadas às ACTs abertas e fechadas; Mercado de trabalho; Oferta de cursos de formação superior; Políticas Públicas.

${ }^{6}$ Orçamento próprio significa que todo o ano o observatório tem um orçamento específico para executar as suas atividades. Recursos próprios estão relacionados a origem dos recursos para executar as pesquisas. Esses recursos podem ser esporádicos, ou seja, não necessariamente pertencentes a um orçamento.
} 
Principal fonte de recursos

Recursos Próprios

Por Pesquisas e Projetos

Editais, Licitações e demais contratações com órgãos públicos

Parcerias com entidades nacionais/internacionais

Total

$\begin{array}{ll}\text { Quantidade } & \text { \% } \\ 21 & 47 \% \\ 13 & 29 \% \\ 9 & 20 \% \\ 2 & 4 \% \\ 45 & 100 \%\end{array}$

Fonte: Autores (2021).

Ao serem questionados se os observatórios detinham sítios eletrônicos, a maioria afirmou possuir sites para divulgação das pesquisas (80\%) (tabela 9). Em relação à utilização das redes sociais, a maioria respondeu que possui (60\%). Dos que utilizam as redes sociais, nossos resultados demonstram que as mais utilizadas são o Facebook e Instagram (49\% cada), seguido por WhatsApp (29\%) e Youtube (20\%).

Tabela 9: Respostas das perguntas 10, 11 e 12 - O Observatório possui site para a divulgação das Pesquisas? O Observatório possui redes sociais? E assinale quais redes sociais possui.

\begin{tabular}{lll}
\hline Possui site para a divulgação & Quantidade & \% \\
\hline Não & 9 & $20 \%$ \\
Sim & 36 & $80 \%$ \\
Total & 45 & $100 \%$ \\
& & \\
Possui redes sociais & Quantidade & $\%$ \\
Não & 27 & $60 \%$ \\
Sim & 18 & $40 \%$ \\
Total & 45 & $100 \%$ \\
& & \\
Rede Social & Quantidade & $\%$ \\
Facebook & 22 & $49 \%$ \\
Instagram & 22 & $49 \%$ \\
WhatsApp & 13 & $29 \%$ \\
Youtube & 9 & $20 \%$ \\
Twitter & 4 & $9 \%$ \\
\end{tabular}




\section{ReBOT UFN}

$\begin{array}{lcc}\text { Website } & 3 & 7 \% \\ \text { LinkedIn } & 2 & 4 \% \\ \text { Blog } & 1 & 2 \%\end{array}$

Fonte: Autores (2021).

Por fim, em relação a relevância da Rede Brasileira de Observatórios de Turismo (RBOT) na consolidação e/ou crescimento dos observatórios, a opção mais positiva se destacou, com $87 \%$ dos representantes alegando que a RBOT possui um papel totalmente relevante ou relevante (tabela 10 ).

Tabela 10: Qual é a relevância da RBOT para a consolidação e/ou crescimento do seu Observatório?

\begin{tabular}{lll}
\hline Relevância da RBOT & Quantidade & $\%$ \\
\hline 5 (totalmente relevante) & 35 & $78 \%$ \\
4 (relevante) & 4 & $9 \%$ \\
3 (neutro) & 5 & $11 \%$ \\
2 (irrelevante) & 1 & $2 \%$ \\
1 (totalmente irrelevante & 0 & 0 \\
Total & 45 & $100 \%$ \\
\hline
\end{tabular}

Fonte: Autores (2021).

Esse resultado demonstra que a RBOT, além de ter como objetivo fomentar o turismo através do planejamento e gerência de base de dados no país, serve de suporte técnico metodológico para os observatórios de turismo em todo território nacional, possibilitando colaboração e cooperação entre os pares.

\section{CONSIDERAÇÕES FINAIS}

A presente pesquisa objetivou realizar o levantamento de informações sobre a composição, a estrutura e a dinâmica das pesquisas realizadas pelos observatórios membros da Rede Brasileira de Observatórios de Turismo. O objetivo foi atingido por meio de análise descritiva de dados coletados por questionários online de 45 observatórios de turismo membros da RBOT. 
A pesquisa reforça a importância do turismólogo na constituição técnica dos observatórios, além de indicar a carência de recursos e de equipe técnica para a realização das atividades de pesquisa. Há também a indicação que, seguindo o ideal de observatórios proposto por Theorga (2016), os observatórios da RBOT são espaços multidisciplinares com profissionais com expertises diferentes.

Os observatórios entrevistados, aparentemente, dão mais atenção aos sítios eletrônicos e estão em menor inserção nas redes sociais, o que pode ser um fator limitador no processo de divulgação e publicização dos dados. Sugerimos, portanto, que os observatórios tenham uma atuação on-line mais intensa, dentro das suas restrições técnicas e de recursos humanos.

Por fim, a presente pesquisa consolida a importância da Rede Brasileira de Observatórios de Turismo ao demonstrar sua relevância na condução das discussões e na definição de questões de interesse de todos os observatórios da rede. A liderança de discussões como a harmonização das metodologias de pesquisa utilizadas pelos observatórios, a representação e a cooperação com diversas instituições nacionais e internacionais de turismo, realização de pesquisas nos diversos estados e municípios, com a participação dos observatórios, são algumas ações e desafios do presente e do futuro da RBOT.

Teoricamente, o presente relatório técnico descritivo consolida a história da Rede Brasileira de Observatórios de Turismo, ao qual carecia de informações concretas, estruturadas e de acesso público para compreensão do processo formativo da rede. Além disto, nossa pesquisa compreende elementos inerentes dos observatórios e fornece base para compreensão dos limites técnicos e estruturais dos mesmos, servindo de base para futuras construções de observatórios em destinos que não possuam conhecimento prévio sobre a constituição e atuação de um observatório.

A presente pesquisa está ciente de suas limitações, sendo primeiramente um estudo survey autorrelatado, o que impossibilita compreendermos se de fato os observatórios estão sendo fidedignos ao responder, apesar de que consideramos tal viés seja pouco importante nessa pesquisa, já que alguns dos membros relataram ser inativos, deste modo, excluídos de nossas análises. 
Além disso, pesquisas tanto quantitativas, quanto qualitativas, são necessárias para adentrar uma infinidade de variáveis apresentada nesse estudo, já que a pesquisa é de caráter descritivo. Frisamos também a necessidade de futuros estudos adentrarem na temática da constituição da RBOT de maneira mais aprofundada, por meio de entrevistas semiestruturadas com participantes de observatórios constituintes desde a construção e das primeiras reuniões da rede. Deste modo, a expectativa é que o estudo possa lançar luz para futuras pesquisas relacionadas aos observatórios de turismo da RBOT, como por exemplo a classificação dos observatórios a partir da natureza das pesquisas realizadas.

\section{Agradecimentos}

Agradecemos ao professor Glauber Santos, docente da Universidade de São Paulo pelas sugestões de análise.

\section{REFERÊNCIAS}

ALVARES, D. F.; SANTOS, S. R.; PERINOTTO, A. R. C. NETWORK OF TOURISM OBSERVATORIES TOWARD A TOURISM INTELLIGENCE: THE CASE OF BRAZIL. ENLIGHTENING TOURISM. A PATHMAKING JOURNAL, v. 10, n. 2, p. 140-178, 2020.

\section{BH EVENTOS. II Seminário Mineiro de Pesquisa e Inovação em Turismo} (SEMPIT). 2017. Disponível em: https://www.bheventos.com.br/evento/11-04-2017-iiseminario-mineiro-de-pesquisa-e-inovacao-em-turismo-sempit. Acesso em: 15 out. 2021.

CACHO, A. et al. Mobile tourist guide supporting a smart city initiative: a Brazilian case study. International Journal of Tourism Cities, 2016.

COELHO, M. F.; MAYER, V. F. Gestão de serviços pós-covid: o que se pode aprender com o setor de turismo e viagens?. Gestão e Sociedade, v. 14, n. 39, p. 3698-3706, 2020. https://doi.org/10.21171/ges.v14i39.3306 
FREITAS, H. et al. O método de pesquisa survey. Revista de Administração da Universidade de São Paulo, v. 35, n. 3, 2000.

HUNG, K.; LAW, R. An overview of Internet-based surveys in hospitality and tourism journals. Tourism Management, v. 32, n. 4, p. 717-724, 2011.

MAIR, J. Exploring air travellers' voluntary carbon-offsetting behaviour. Journal of Sustainable Tourism, v. 19, n. 2, p. 215-230, 2011.

MARQUES, O. Rede de Observatórios de Turismo é criada em Encontro Paranaense. Revista Portal Eventos. 2017. Disponível em: https://www.revistaeventos.com.br/blog/Turismo-e-Eventos/Rede-de-Observatorios-deTurismo-e-criada-em-Encontro-Paranaense/41402. Acesso em: 15 out. 2021.

MARQUES, O. R. B.; MONTEIRO, J. E. D.; SOUZA NETO, V. R. Rede Brasileira de Observatórios de Turismo. 2021. Disponível em: http://observatoriodoturismo.uff.br/?page_id=777. Acesso em: 02 jul. 2021.

MAYER, V. F.; COELHO, M. F. Sonhos interrompidos: memórias e emoções de experiências de viagem durante a propagação da Covid-19. Revista Brasileira de Pesquisa em Turismo, v. 15, 2021. https://doi.org/10.7784/rbtur.v15i1.2192

MEDAGLIA, J.; SILVEIRA, C. E. Sondagem empresarial dos impactos da covid-19 no setor de turismo no paraná: o observatório de turismo do Paraná e o protagonismo da informação. Revista Turismo \& Cidades, v. 2, p. 153-171, 2020.

MONDO, T. TOURQUAL: Proposta de um modelo de avaliação da qualidade de serviços em atrativos turísticos (Tese de Doutorado, 399 p.) Florianópolis, SC, 2014.

MTUR, Ministério do Turismo. Plano nacional de Turismo 2018-2022: mais emprego e renda para o Brasil. Disponível em: http://regionalizacao.turismo.gov.br/images/PNT_2018-2022.pdf. 2018.

OMT, Organização Mundial do Turismo. UNWTO INTERNATIONAL NETWORK OF SUSTAINABLE TOURISM OBSERVATORIES. s.a.. Disponível em: https://www.unwto.org/sustainable-development/unwto-international-network-ofsustainable-tourism-observatories. Acesso em: 13 out. 2021. 
RBOT, Rede Brasileira de Observatórios de Turismo. $4^{\circ}$ Encontro da Rede Brasileira dos Observatórios de Turismo. Parte II Pesquisa em Rede: Entrelaçamentos do Turismo Brasileiro. Foz do Iguaçu. 2018.

- Carta de Curitiba de Apoio à Criação da Rede Brasileira de

Observatórios de Turismo. In: Encontro Paranaense de Observatórios de Turismo.

Curitiba - Paraná. 3 p. 2017.

. Memória - 2º Encontro da RBOT. Foz do Iguaçu. 2018.

. Proposta De Parceria Para Realização De Evento II Encontro Da Rede

Brasileira De Observatórios De Turismo. Foz do Iguaçu. 2018.

SECULT-MG, Secretaria de Estado de Cultura e Turismo de Minas Gerais. Setur realiza $1^{\text {a }}$ Reunião de Observatórios de Turismo. Disponível em: https://www.secult.mg.gov.br/noticias-artigos/1265-setur-realiza-1o-reuniao-deobservatorios-de-turismo. Acesso em: 15 out. 2021.

SIGALA, M. New technologies in tourism: From multi-disciplinary to anti-disciplinary advances and trajectories. Tourism management perspectives, v. 25, p. 151-155, 2018.

THEORGA, A. B. Os observatórios de turismo no Brasil; orientador José Aroudo Mota - Brasília, Dissertação (Mestrado Profissional em Turismo) -UnB, 2016.

XIANG, Z. et al. Adapting to the internet: trends in travelers' use of the web for trip planning. Journal of travel research, v. 54, n. 4, p. 511-527, 2015.

XIANG, Z. From digitization to the age of acceleration: On information technology and tourism. Tourism management perspectives, v. 25, p. 147-150, 2018. 